\title{
Escala de Funcionamento Psicológico Positivo: Adaptação e Estudos Iniciais de Validação em Universitários Portugueses
}

\author{
Positive Psychological Functioning Scale: Adaptation and Initial Validation Studies \\ with Portuguese College Students
}

\author{
Ema Patrícia Oliveira ${ }^{1}$, María Dolores Merino ${ }^{2}$, Jesus Privado ${ }^{3}$ e Leandro S. Almeida ${ }^{4}$
}

\begin{abstract}
Resumo
A relevância da investigação centrada no bem-estar tem sido reforçada ao longo das últimas décadas, em especial após a emergência da Psicologia Positiva. Neste âmbito, é necessário investir na construção e validação de instrumentos adequados para avaliar as dimensões inerentes a um funcionamento psicológico saudável. Este estudo pretende adaptar e validar a Escala de Funcionamento Psicológico Positivo (EFPP; Merino \& Privado, 2015) junto de uma amostra de universitários portugueses. Participaram no estudo 1131 estudantes de duas universidades, com média de idade de 19.6 anos. Além da EFPP, para efeitos de validade de critério foi aplicada a Satisfaction With Life Scale (SWLS; Diener et al., 1985). Os resultados evidenciam boas propriedades psicométricas da EFPP, tanto ao nível da fiabilidade como da validade. Dada a escassez de instrumentos de avaliação do funcionamento positivo que se tenham revelado satisfatórios no nosso país, a utilização desta escala poderá ser recomendada, particularmente com jovens universitários.
\end{abstract}

Palavras-chave: bem-estar psicológico, psicologia positiva, avaliação, ensino superior

\begin{abstract}
In the last decades research focused on well-being has been prominent, especially with the emergence of Positive Psychology. In this context, it is necessary to invest in the construction and validation of appropriate instruments to assess the dimensions of a healthy psychological functioning. This study aims to adapt and validate the Positive Psychological Functioning Scale (EFPP; Merino \& Privado, 2015) with a sample of Portuguese university students. A total of 1131 students from two universities participated in the study, with an average age of 19.6 years. In addition to EFPP, Satisfaction With Life Scale (SWLS; Diener et al., 1985) was used for criterion validity. Results showed good psychometric properties of EFPP, both in terms of reliability and validity. Given the scarcity of positive functioning assessment instruments that have proved to be satisfactory in our country, the use of this scale may be recommended, particularly with young university students.
\end{abstract}

Keywords: psychological well-being, positive psychology, assessment, higher education

\footnotetext{
${ }^{1}$ Doutoramento em Psicologia. Professora Auxiliar, Universidade da Beira Interior. Faculdade de Ciências Sociais e Humanas, Estrada do Sineiro, 6200-209 Covilhã, Portugal. Tel.: +351275329165. E-mail: ema@ubi.pt

2 Doutoramento em Psicologia. Professor Associado, Universidad Complutense de Madrid. Facultad de Psicología, Universidad Complutense de Madrid, Campus de Somsaguas. 28223 Madrid, España. Tel.: +34913943234. E-mail: lolamerino@psi.ucm.es

${ }^{3}$ Doutoramento em Psicologia. Professor Auxiliar, Universidad Complutense de Madrid. Facultad de Psicología, Universidad Complutense de Madrid, Campus de Somsaguas. 28223 Madrid, España. Tel.: +34 91394 30 80. E-mail: jesus.privado@pdi.ucm.es

${ }^{4}$ Doutoramento em Psicologia. Professor Catedrático, Universidade do Minho. Universidade do Minho, Campus de Gualtar, 4715 Braga-Portugal. Tel.: +351253604255. E-mail: leandro@ie.uminho.pt
} 


\section{Introdução}

$\mathrm{O}$ estudo sobre o bem-estar e a felicidade no seio da psicologia é relativamente recente. A sua origem remonta à década de 60 do século passado e à influência das perspetivas humanistas e construtivistas na emergência da Psicologia Positiva. Neste quadro, de um enfoque na doença ou na psicopatologia, esta abordagem centra-se nas condições promoras do bem-estar, da saúde mental e do crescimento pessoal, enfatizando os aspetos saudáveis do ser humano, o seu potencial de desenvolvimento, os seus recursos e virtudes (Diener, 1984; Ryff, 1989; Seligman \& Csikszentmihalyi, 2000). Entende-se, assim, que para compreender o bem-estar e promover condições facilitadoras da vida não basta compreender a infelicidade, nem tão pouco eliminar as condições debilitantes vivenciadas (Seligman, 2012). A investigação psicológica passa, então, a investir em aspetos como a sabedoria, a criatividade, o otimismo, a responsabilidade, a competência, entre outros (Novo, 2005). Nesta linha, importa destacar a associação entre as características individuais positivas em populações em idade escolar e vários fatores protetores e promotores da saúde, rendimento académico e diversos interesses vocacionais (Raimundi, Molina, HernándezMendo, \& Schmidt, 2017). Ao nível do ensino superior, por exemplo, alguns estudos com amostras portuguesas têm apontado correlações positivas entre o bem-estar psicológico, ou algum dos componentes do funcionamento psicológico positivo, tais como o otimismo, e a adaptação psicossocial e académica dos estudantes (Laginha, 2016; Monteiro, 2008; Porta-Nova, 2009).

Numa perspetiva mais alargada e histórica, o estudo sobre o bem-estar tem as suas raízes em duas tradições filosóficas gregas distintas, mas em estreita ligação: a da hedonia e a da eudaimonia. A perspetiva hedonista define o bem-estar em termos de obtenção de prazer e evitamento de sofrimento, dando maior destaque ao bem-estar afetivo a curto-prazo e menor aos desafios duradouros da vida, tais como estabelecer relações interpessoais satisfatórias ou desenvolver um sentido de autorrealização (Ryff, 1989; Waterman, 1984). A maioria da investigação em psicologia que se insere nesta abordagem tem-se baseado no bem-estar subjetivo, um constructo que inclui três componentes: satisfação com a vida, presença de emoções positivas e ausência de afeto negativo. Este enfoque nas emoções não engloba aspetos também importantes do bem-estar como a autonomia e o crescimento pessoal (Ryan \& Deci, 2001; Ryff, 1989).

Por outro lado, a abordagem eudaimónica, decorrente do pensamento aristotélico, foca-se no significado e na autorrealização, definindo o bemestar em termos do grau de funcionamento pleno do ser humano. Para Aristóteles, o bem-estar é alcançado através da rentabilização e do desenvolvimento do melhor em si próprio, de acordo com os princípios pessoais mais profundos (Huta \& Ryan, 2010). Desta forma, a felicidade é vista como o produto de uma vida com propósito, em direção à autorrealização e a ideais de crescimento pessoal, de aperfeiçoamento contínuo e de atualização do potencial humano, ou seja, do seu verdadeiro self ("daimón") (Ryan \& Deci, 2001). Em consonância com esta perspetiva, as medidas desenvolvidas para avaliar o bem-estar psicológico, no geral, são muldimensionais. Uma das mais conhecidas e utilizadas é a Scales of Psychological Well-Being de Ryff (1989). Esta autora, baseando-se no trabalho sobre a saúde mental de Jahoda (1958), entre outras conceções e modelos teóricos no âmbito da psicologia, identificou seis dimensões de bem-estar que se agrupam num fator de segunda ordem, a que denominou Bem-Estar Psicológico. Tais dimensões foram: aceitação de si (manter uma atitude positiva face a si mesmo e ao seu passado), autonomia (ter um sentido de autodeterminação; uma ação dirigida por valores pessoais; resistir a pressões e convenções sociais), domínio do meio (gerir eficazmente a sua vida e o mundo ao seu redor; desempenhar um papel ativo para alcançar o que necessita e deseja do meio ambiente), relações positivas com os outros (construir relações interpessoais de qualidade; preocupar-se com o bem-estar dos cidadãos), propósito na vida (acreditar que a vida tem um propósito $\mathrm{e}$ significado; estabelecer e lutar por objetivos pessoais que dão sentido e direção à vida) e crescimento pessoal (lutar por um desenvolvimento pessoal contínuo e de concretização do seu potencial; manter-se aberto à experiência; identificar desafios em diferentes 
circunstâncias). Encontram-se atualmente disponíveis diferentes versões das Escalas de Bem-Estar Psicológico (EBEP), adaptadas, traduzidas e/ou validadas em diferentes países (e.g. Alemanha, Austrália, Brasil, Canadá, China, Espanha, Grécia, Holanda, Itália, Suécia, etc.), sendo provavelmente o instrumento com uma utilização mais ampla na avaliação do bem-estar psicológico, a nível internacional. Uma síntese sobre o desenvolvimento destes trabalhos em diferentes países é apresentada por Machado e Bandeira (2012), no seu estudo sobre a validação das EBEP com uma amostra de estudantes universitários brasileiros.

Em Portugal, a adaptação das EBEP foi feita, originalmente, por Novo, Duarte-Silva e Peralta (1997), seguindo-se um outro trabalho de adaptação da autoria de Ferreira e Simões (1999). Em ambos os estudos são reportados resultados satisfatórios ao nível da consistência interna e uma hierarquização semelhante das médias nas diferentes escalas, nomeadamente, valores superiores na dimensão crescimento pessoal e, por ordem decrescente, propósito na vida, relações positivas com os outros, aceitação de si, autonomia e domínio do meio. Também com amostras de estudantes do ensino superior se tem verificado esta ordenação nos valores médios nas seis dimensões, nomeadamente nos contextos português e brasileiro (Machado, 2010; Soares, 2014).

Não obstante, ainda que o modelo de Ryff esteja bem articulado a nível teórico (Ryff, 1989; Ryff \& Singer, 2008), alguns investigadores não conseguiram replicar a validade fatorial da escala manifestando, em consequência, algumas dúvidas acerca da estrutura latente do modelo (Abbott, Ploubidis, Huppert, Kuh, \& Croudace, 2010; Stones, Worobetz, \& Brink, 2011). Em Portugal, por exemplo, têm sido encontradas fragilidades psicométricas em alguns itens da escala, assim como uma estrutura interna diferente da versão original, e algo redundante (Ferreira \& Simões, 1999), sendo os resultados divergentes de estudo para estudo, mesmo quando consideramos apenas amostras de estudantes universitários (Lopes, 2015; Ramos, 2016; Soares, 2014). Torna-se, assim, evidente a necessidade de clarificar melhor a estrutura fatorial do instrumento. Noutros países, como em Espanha (Rodríguez-Carvajal, Díaz,
Moreno-Jiménez, Blanco, \& Van Dierendonck, 2010), foi proposta a ampliação do modelo, acrescentando mais duas dimensões, nomeadamente recursos internos e vitalidade. Por sua vez, seguindo o enfoque eudaimónico do bemestar, Merino e Privado desenvolveram, em 2015, a escala de Funcionamento Psicológico Positivo (EFPP), com base no conceito de "recurso" proposto por Hobfoll (1989; 2002). Esta escala é formada por onze recursos psicológicos, entendidos como: "Aquellas características positivas de la personalidad que: 1) tienen valor en sí mismas, pues se asocian a resultados favorables para el individuo; 2) permiten una mejor adaptación al entorno, al cambio, y promueven el progreso individual hacia la consecución de metas personales y a la satisfacción de necesidades; 3) son maleables por el entorno, pueden ser aprendidas, aunque también son estables" (Merino \& Privado, 2015, p. 45).

Considerando as afirmações anteriores, e a partir da análise da literatura, os autores selecionaram onze recursos psicológicos fundamentais para o bem-estar e a saúde psicológica. Tais recursos são: autoestima (avaliação global positiva acerca de si mesmo e da sua vida), resiliência (capacidade para superar a adversidade e crescer com a experiência), curiosidade (interesse em descobrir e adquirir novas aprendizagens e conhecimentos), otimismo (crença generalizada e estável de um futuro positivo, capacidade para ver o lado positivo das coisas), autonomia (autodeterminação, autogoverno, autoconfiança), vitalidade (sentir-se ativo, com entusiasmo e energia), domínio do meio (capacidade para gerir e monitorizar eficazmente as atividades e exigências da vida diária), propósito na vida (estabelecer e procurar alcançar objetivos pessoais que dão sentido e orientação à vida), humor (encontrar sentido de humor em muitas e variadas situações), desfrute (identificar e desfrutar de experiências diárias positivas), e criatividade (originalidade, flexibilidade cognitiva).

No seguimento dos trabalhos anteriores, este estudo tem como objetivo a adaptação e validação da escala de Funcionamento Psicológico Positivo junto de estudantes portugueses do ensino superior. A par dos cuidados na tradução e adequação cultural do conteúdo dos itens - 
facilitadas pela proximidade linguística cultural entre Espanha e Portugal -, descrevem-se as qualidades métricas em termos de precisão e validade dos resultados.

\section{Método}

\section{Participantes}

A amostra avaliada foi composta por 1131 estudantes universitários portugueses, de duas instituições públicas no norte e centro do país. A média de idade foi de 19.6 anos $(D P=3.47$ anos), oscilando entre $17(n=112)$ e 67 anos $(n=1)$. Os alunos frequentavam o $1^{\circ}(n=768 ; 67.9 \%)$ e o $3^{\circ}$ anos de diferentes cursos (deliberadamente neste estudo decidimos trabalhar com alunos no início e no final da graduação). A maioria dos alunos eram do sexo feminino ( $n=647 ; 57.2 \%)$. Considerando que em Portugal a colocação dos alunos no ensino superior é feita tomando a média de acesso (sistema de numerus clausus), nesta amostra $67.1 \%(n=759)$ dos alunos referiram que estavam a frequentar o curso que selecionaram em primeira opção e $59.1 \%$ ( $n=668)$ a universidade de primeira escolha.

\section{Instrumentos}

A Escala de Funcionamento Psicológico Positivo (EFPP; Merino \& Privado, 2015) é formada por 33 itens com um formato de resposta Likert (em que 1=Discordo Totalmente e 5=Concordo Totalmente), agrupados em 11 subescalas que correspondem a diferentes recursos psicológicos. As subescalas, compostas cada uma por três itens, são as seguintes: Autoestima (e.g., "Gosto da minha forma de ser"), Resiliência (e.g., "Não me rendo facilmente perante as dificuldades da vida"), Curiosidade (e.g., "Muitas coisas da vida despertam a minha curiosidade e interesse"), Otimismo (e.g., "Creio que o futuro me trará mais coisas boas que más"), Autonomia (e.g., "As decisões importantes da minha vida, para o melhor ou para o pior, foram tomadas por mim"), Vitalidade (e.g., "Sou uma pessoa cheia de energia"), Domínio do Meio (e.g., "Concilio adequadamente a minha vida académica, social e pessoal”), Propósito na Vida (e.g., "Estou completamente centrado/a em conseguir atingir os objetivos da minha vida"), Humor (e.g., "Tento encontrar humor em qualquer situação"), Desfrute (e.g., "Sei desfrutar das pequenas coisas que a vida me oferece em cada dia") e Criatividade (e.g., "Sou capaz de ver as coisas a partir de pontos de vista completamente diferentes").

Estando a tradução dos itens para português facilitada pela proximidade linguística e cultural da versão original em espanhol, mesmo assim foi necessário proceder à "reflexão falada" com grupos focais de estudantes das duas universidades presentes na amostra. O conteúdo de alguns itens em português foi modificado no contacto com os autores da versão original por forma a aproximar os itens da dimensão avaliada (em anexo apresentamos a versão final da escala em língua portuguesa).

Importa salientar que a versão original da escala (Merino \& Privado, 2015) apresentou boas propriedades psicométricas, tanto em termos de precisão como de validade (convergente, descriminante e preditiva). Ao nível da precisão, o coeficiente alfa de Cronbach na pontuação total da escala foi de .91 , situando-se entre .70 e .80 nas subescalas, com exceção da Autonomia $(\alpha=.66)$. Além disso, a EFPP foi adaptada com êxito à população mexicana (Merino, Privado, \& Gracia, 2015), onde também se verificaram propriedades psicométricas adequadas - as subescalas Desfrute e Resiliência apresentaram menores valores em termos de consistência interna ( $\alpha=.53$ e $\alpha=.56$, respetivamente), no entanto a pontuação total da escala, que inclui as dimensões referidas, apresentou uma precisão adequada $(\alpha=.91)$, e os valores das restantes subescalas apresentaram um coeficiente alfa de Cronbach acima de .66, sendo o mais elevado na subescala Propósito na Vida $(\alpha=.84)$. No México, tal como em Espanha, as análises de equações estruturais realizadas demonstraram que os 11 recursos avaliados se agrupavam num fator de segunda ordem, denominado Funcionamento Psicológico Positivo (Merino \& Privado, 2015). Os coeficientes de precisão e de validade da versão portuguesa são apresentados mais à frente neste artigo.

Para a análise de validade dos resultados, utilizou-se neste estudo a escala Satisfaction With Life Scale (SWLS; Denier, Emmons, Larsen, \& Griffin, 1985), na sua versão portuguesa adaptada por Martins, Araújo e Almeida (2015). Esta escala 
é constituída por cinco itens, sendo as respostas fornecidas numa escala de tipo Likert, variando entre 1 (totalmente em desacordo) e 7 (totalmente de acordo). Trata-se de um instrumento amplamente utilizado para a avaliação do bemestar subjetivo, mais concretamente a satisfação com a vida. Os estudos de validação realizados em Portugal com a SWLS têm reportado níveis elevados de precisão e uma estrutura interna que aponta para a unidimensionalidade dos itens (Martins, Araújo, \& Almeida, 2015; Neto, Barros, \& Barros, 1990; Simões, 1992). A consistência interna dos itens desta escala no presente estudo situou-se em .81 .

\section{Procedimento}

A recolha de dados foi realizada ao longo dos anos letivos de 2015/2016 e 2016/2017. No primeiro ano letivo os instrumentos foram aplicados em sala de aula, em tempo cedido pelos professores; no segundo, os questionários foram preenchidos no momento da matrícula na universidade. Em ambas as situações, os estudantes foram informados dos objetivos do estudo e da confidencialidade dos resultados, dando o seu consentimento informado. Os dados foram analisados com recurso ao software IBM SPSS e AMOS, versão 23.0.

Para a análise fatorial confirmatória utilizamos o método de estimação maximum likelihood (MLE) e como índices de ajuste a divisão do qui-quadrado pelos graus de liberdade $\left(\chi^{2} / d f\right)$ sendo que Iacobucci (2009) sugere valores abaixo de 3, o CFI (Comparative Fit Index), GFI (Goodness Fit Index), AGFI (Adjusted Goodness of Fit Index) e NFI (Normed Fit Index) com valores >.90 (Hu \& Bentler, 1999), e o RMSEA (Root Mean Square Error of Approximation) com valores menores que .08 (Browne \& Cudeck, 1993). Para o coeficiente alfa de Cronbach assumimos valores iguais ou superiores a .70, ainda que este coeficiente possa estar negativamente afetado pelo número reduzido de itens nas subescalas (Nunnally \& Bernstein, 1994).

\section{Resultados}

No Quadro 1 apresenta-se a distribuição de resultados nas 11 dimensões da EFPP, bem como a pontuação global. Igualmente se apresenta a distribuição de resultados na SWLS. Nesta apresentação inclui-se a média e desvio-padrão, os coeficientes de assimetria e curtose, bem como o alfa de Cronbach. Tomando as médias, verificamos um valor mais baixo na dimensão Domínio do Meio e valores mais elevados quando os alunos se autoavaliam ao nível do Propósito na Vida, Humor e Autoestima.

Quadro 1. Distribuição dos resultados nas subescalas e no total da EFPP e na SWLS (n=1131)

\begin{tabular}{lccccccc}
\hline & & & & & \multicolumn{3}{c}{ Alfa de Cronbach } \\
\cline { 6 - 8 } Dimensões & $M$ & $D P$ & Assim. & Curt. & Portugal & Espanha & México \\
\hline Autoestima & 12.50 & 1.94 & -.67 & .45 & .79 & .76 & .79 \\
Resiliência & 12.29 & 1.70 & -.45 & .59 & .71 & .71 & .56 \\
Curiosidade & 11.89 & 1.62 & -.05 & -.04 & .64 & .72 & .67 \\
Otimismo & 11.36 & 1.98 & -.30 & .07 & .67 & .75 & .72 \\
Autonomia & 12.17 & 1.63 & -.42 & .68 & .60 & .66 & .69 \\
Vitalidade & 11.67 & 1.93 & -.49 & .44 & .77 & .66 & .80 \\
Dom. Meio & 11.07 & 1.87 & -.59 & .97 & .61 & .71 & .66 \\
Prop. Vida & 12.84 & 1.61 & -.65 & .94 & .77 & .71 & .84 \\
Humor & 12.69 & 1.78 & -.56 & .14 & .75 & .73 & .77 \\
Desfrute & 11.53 & 1.65 & -.30 & .28 & .63 & .72 & .53 \\
Criatividade & 11.82 & 1.48 & -.04 & .00 & .60 & .80 & .68 \\
FPP Global & 12.0 & 1.24 & -.28 & .74 & .90 & .91 & .91 \\
SWLS & 26.86 & 4.76 & -.79 & .67 & .81 & & \\
\hline
\end{tabular}


Os índices de assimetria e curtose são adequados. West, Finch e Curran (1995) referem que, com amostras grandes como a aqui utilizada, valores de assimetria inferiores a 2 em valor absoluto e inferiores a 7 de curtose podem ser adequados à utilização da máxima verosimilhança como procedimento de estimativa. Como se pode ver, a maioria dos itens apresentam assimetria negativa, ou seja, trata-se de uma amostra que se autoavalia como apresentando um funcionamento psicológico bastante alto, o que é esperado em amostras retiradas de populações não clínicas.

Relativamente aos coeficientes de fiabilidade das diferentes subescalas da EFPP, todas elas apresentam valores superiores a .60, o que se pode considerar positivo (sobretudo quando formadas por apenas 3 itens). No seu conjunto, a EFPP apresenta um alfa de .90, considerado muito satisfatório (Nunnally \& Bernstein, 1994). De acrescentar, que a dimensão Domínio do Meio na versão original incluía um item formulado pela negativa (o único na escala), que foi testado ao mesmo tempo que uma sua versão pela positiva, ficando o alfa melhor neste último caso, pelo que todos os itens da versão portuguesa da EFPP são formulados pela positiva.

Em termos de validade, realizou-se uma análise fatorial confirmatória sobre as 11 subescalas da EFPP, com o objetivo de verificar se os resultados se ajustavam a um único fator latente. Na Figura 1 apresenta-se o modelo submetido a análise, assinalando os loadings de cada uma das 11 dimensões no fator geral. Como se pode verificar, a subescala Humor apresenta-se menos associada a este fator geral de funcionamento psicológico positivo (.48), e o contrário ocorre com as subescalas Vitalidade (.77) e Desfrute (.77), o que nos parece interessante pois traduzem duas características centrais do funcionamento psicológico positivo (disposição para aproveitar o presente e energia ou vigor implementado nessa prossecução).

No Quadro 2 apresentam-se os índices de ajuste da análise fatorial confirmatória, tomando o modelo inicial e o modelo modificado depois de se introduzirem algumas alterações sugeridas pelo software. Estas modificações prenderam-se exclusivamente com a correlação entre os erros E1 e E5, E2 e E8, E3 e E10, e E3 e E11. A capacidade de lidar bem com a novidade e a

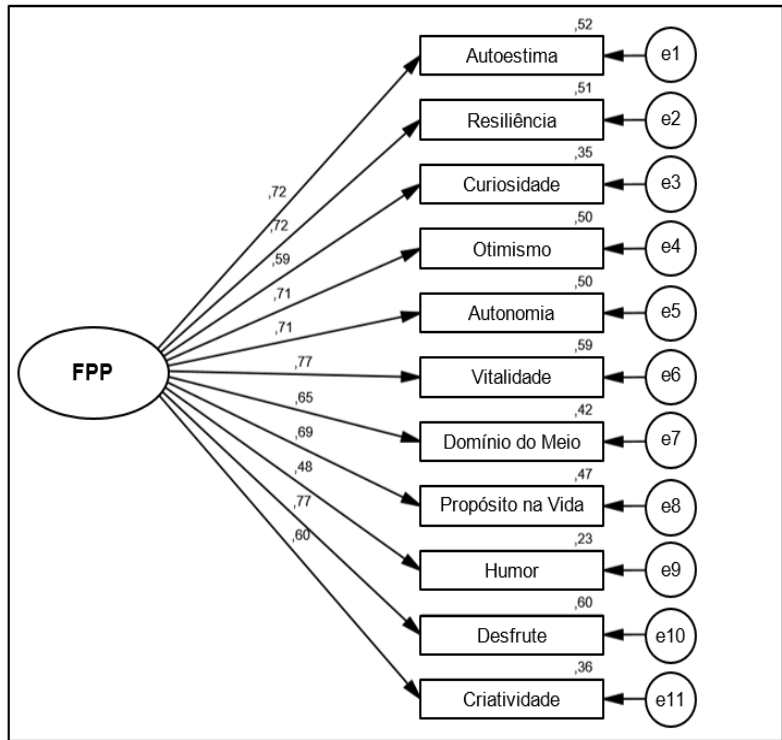

Figura 1. Estrutura interna das escalas da EFPP

abertura à experiência poderão explicar as correlações entre Curiosidade e Desfrute, assim como Curiosidade e Criatividade. Ao mesmo tempo, a ligação entre Resiliência e Propósito na Vida tem sido enfatizada, por exemplo no seio das abordagens existenciais, em especial a procura de sentido face a situações de adversidade (Ryff, 2016). Considera-se que a experiência de perda de sentido e propósito na vida torna os indivíduos mais vulneráveis ao sofrimento e, nesta linha, que a resiliência permite operacionalizar algumas das consequências de se encontrar o sentido da vida (Frankl, 2004). A relação entre resiliência e propósito na vida tem também sido corroborada pela investigação (e.g. Nygren, Aléx, Jonsén, Gustafson, Norberg, \& Lundman, 2005). Por outro lado, sendo esta amostra constituída essencialmente por jovens adultos, entende-se a correlação encontrada entre Autoestima e Autonomia. Por fim, os índices obtidos mostram um ajuste do modelo aos dados bastante aceitável, o que confirma que a escala apresenta um fator geral, que se identifica com o funcionamento psicológico positivo.

Quadro 2. Índices de ajuste do modelo

\begin{tabular}{lcccccc}
\hline Índices & $\mathrm{Chi}^{2} / \mathrm{gl}$ & GFI & AGFI & NFI & CFI & RMSEA \\
\hline $\begin{array}{l}\text { Modelo } \\
\text { Teórico }\end{array}$ & 12.096 & .913 & .869 & .906 & .913 & .099 \\
$\begin{array}{l}\text { Modelo } \\
\text { Modificado }\end{array}$ & 7.54 & .952 & .921 & .945 & .951 & .078 \\
\hline
\end{tabular}


Quadro 3. Correlações entre as dimensões da EFPP e a SWLS

\begin{tabular}{|c|c|c|c|c|c|c|c|c|c|c|c|c|}
\hline & $\mathrm{AE}$ & $\mathrm{R}$ & Cur & $\mathrm{O}$ & Aut & $\mathrm{V}$ & DM & PV & $\mathrm{H}$ & $\mathrm{D}$ & Cri & FPPG \\
\hline Autoestima (AE) & - & & & & & & & & & & & \\
\hline Resiliência (R) & .52 & - & & & & & & & & & & \\
\hline Curiosidade (Cur) & .33 & .42 & - & & & & & & & & & \\
\hline Otimismo (O) & .52 & .50 & .35 & - & & & & & & & & \\
\hline Autonomia (Aut) & .59 & .56 & .37 & .51 & - & & & & & & & \\
\hline Vitalidade (V) & .58 & .50 & .44 & .58 & .49 & - & & & & & & \\
\hline Domínio Meio (DM) & .44 & .43 & .34 & .51 & .42 & .54 & - & & & & & \\
\hline Prop. Vida (PV) & .54 & .62 & .45 & .42 & .54 & .49 & .44 & - & & & & \\
\hline Humor $(\mathrm{H})$ & .30 & .26 & .32 & .38 & .32 & .44 & .30 & .22 & - & & & \\
\hline Disfrute (D) & .53 & .51 & .54 & .56 & .49 & .64 & .53 & .47 & .43 & - & & \\
\hline Criatividade (Cri) & .36 & .46 & .50 & .39 & .45 & .41 & .39 & .41 & .30 & .46 & - & \\
\hline FPP Global (FPPG) & .74 & .74 & .64 & .74 & .73 & .79 & .69 & .71 & .55 & .79 & .64 & \\
\hline SWLS & .60 & .41 & .35 & .45 & .44 & .52 & .45 & .49 & .23 & .51 & .28 & 61 \\
\hline
\end{tabular}

Nota. Todas as correlações são estatisticamente significativas para $p<.01$

No Quadro 3 apresentam-se as correlações entre as subescalas e os totais da EFPP e da SWLS, para efeitos de validade convergente. Os resultados demonstram correlações positivas estatisticamente significativas entre todas as subescalas e o total da EFPP, com valores médioaltos na maioria dos casos. O contruto geral FPP apresenta a correlação mais elevada (.61) com a medida de bem-estar subjetivo utilizada, a SWLS. As diferentes subescalas do FPP seguem o mesmo padrão que a medida global, mas com correlações mais baixas, uma vez que cada uma delas integra a escala global. Destacam-se, neste caso, as subescalas Curiosidade, Humor e Criatividade, traduzindo especificidades dos constructos avaliados. Estes coeficientes estão em conformidade com os loadings apresentados na Figura 1, onde se podem observar índices mais baixos para estas três subescalas no fator geral de Funcionamento Psicológico Positivo.

\section{Considerações finais}

À luz da Psicologia Positiva, importa não reduzir a investigação e a intervenção psicológicas às vertentes psicopatológicas do desenvolvimento e do comportamento humano, as quais acentuam um foco remediativo dessa mesma intervenção. Ao considerarmos a relevância de recursos e virtudes do ser humano, adquirimos uma perspetiva mais ampla sobre o bem-estar psicológico e as condições facilitadoras do seu desenvolvimento e promoção. Neste sentido, importa investir na construção e validação de instrumentos suscetíveis de avaliarem as dimensões psicológicas inerentes a este constructo.
Um dos modelos que dá suporte à avaliação do bem-estar psicológico decorre dos trabalhos de Carol Ryff (1989), assumindo seis componentes do funcionamento psicológico positivo (aceitação de si, autonomia, domínio do meio, relações positivas com os outros, propósito na vida e crescimento pessoal). Nesta mesma orientação, e acrescentando dimensões associadas a recursos psicológicos, apresentamos a Escala de Funcionamento Psicológico Positivo (EFPP; Merino \& Privado, 2015) composta por 11 dimensões.

Centrando-se este artigo na adaptação e validação da EFPP para a população universitária portuguesa, os resultados da análise fatorial confirmatória sugerem uma adequação do modelo teórico de partida, apontando a convergência das 11 dimensões da escala num fator geral, tendo-se replicado assim a estrutura fatorial encontrada com as populações espanhola (Merino \& Privado, 2015) e mexicana (Merino, Privado, \& Gracia, 2015). Entre estes fatores, importa destacar os loadings inferiores das subescalas Humor, Curiosidade e Criatividade face às subescalas Desfrute e Vitalidade. Aquelas três dimensões parecem mais associadas à novidade das situações e abertura à experiência, enquanto as duas últimas têm mais a ver com a disposição para aproveitar positivamente as situações e nelas investir de forma ativa, com energia ou vigor (Ryan \& Frederick, 1997).

A dimensionalidade da EFPP sai reforçada pelos coeficientes de consistência interna de cada uma das onze dimensões, sendo certo que os valores se podem considerar moderados nalgumas 
delas, em virtude de um número reduzido de itens em presença ( 3 itens por subescala). $\mathrm{O}$ mesmo se verificou nos estudos de validação da EFPP em Espanha e no México. Apesar de os resultados não serem tão elevados como os que se verificaram com a população espanhola, onde os valores mais baixos se situaram em .66 (subescalas Autonomia e Curiosidade) (Merino \& Privado, 2015), eles aproximam-se bastante dos verificados no México, onde a amostra também foi constituída por estudantes de licenciatura do ensino superior (Merino, Privado, \& Gracia, 2015). Esta conformidade de resultados verificouse tanto no valor global da EFPP (.90 em Portugal e .91 no México) como nas subescalas em geral, sendo que na amostra mexicana os coeficientes de consistência interna mais baixos não chegaram a 60 (nas subescalas Desfrute e Resiliência).

Ao nível da validade convergente, analisando as correlações entre as subescalas da EFPP e a pontuação global desta escala com a Satisfaction With Life Scale, observamos valores moderados ou elevados de correlação. Estes valores são mais altos tomando a escala global e a subescala Autoestima, parecendo-nos esta última dimensão particularmente relevante nas perceções pessoais de bem-estar. Este dado expressa que pessoas com elevada autoestima tendem a relatar níveis mais elevados de felicidade (Holder \& Klassen, 2009). Por sua vez, as subescalas Humor e Criatividade apresentam correlações mais baixas, traduzindo especificidades pessoais, provavelmente não generalizáveis a toda a amostra, e que poderão não ser essenciais às perceções de bem-estar dos indivíduos.

Finalmente, importa destacar algumas limitações do presente estudo e implicações para futuras pesquisas na área. Uma das limitações prende-se com a utilização de uma amostra de apenas duas universidades, não abarcando a diversidade de instituições de ensino superior universitário e politécnico ou público e privado em Portugal. Por outro lado, ainda que tenham sido incluídos estudantes no início e no final da sua graduação, a maioria dos participantes frequentava $\mathrm{o} 1^{\mathrm{o}}$ ano, podendo existir alguma especificidade associada a este grupo particular de estudantes que experiencia vivências próprias de um processo de transição e adaptação ao ensino superior. Ainda, predomina na amostra um grupo etário de estudantes entre os 17 e os 21 anos, ou seja numa fase de desenvolvimento psicossocial definida por "adultez-emergente" (Arnett, 2016; Ferreira \& Jorge, 2008; Soares, 2003), sendo importante em futuros estudos incluir estudantes mais velhos, nomeadamente quando as instituições se abrem progressivamente a novos públicos. Nesta altura, assumindo uma maior diversidade de estudantes, importa verificar se os itens da escala são representativos e suficientemente abrangentes das dimensões psicológicas avaliadas, mais ainda quando cada dimensão é formada por apenas três itens. Ao mesmo tempo, a validade convergente foi apenas estimada tomando uma medida de bem-estar subjetivo, o que não potencializa uma análise da diversidade de dimensões presentes na EFPP. Em termos futuros, seria pertinente considerar outras escalas avaliando dimensões psicológicas positivas, assim como incluir indicadores mais diretos do bem-estar e do funcionamento psicológico positivo, nomeadamente classificações académicas ou redes de suporte social, a título de exemplo. Entendendo-se que o ensino superior coloca desafios aos estudantes e que estes desenvolvem as suas competências académicas, profissionais e psicossociais no decurso da formação superior, seria importante estudar no futuro como as perceções de bem-estar psicológico contribuem para o sucesso destas trajetórias desenvolvimentais e, inclusive, como tais perceções aumentam quando o percurso formativo é pautado por níveis superiores de sucesso académico. Em particular, estando os países e as instituições de ensino superior preocupadas com as taxas de abandono dos seus estudantes, importará no futuro verificar quanto o bem-estar psicológico se constitui em fator promotor da permanência, investimento e conclusão dos cursos.

\section{Referências}

Abbott, R. A., Ploubidis, G. B., Huppert, F.A., Kuh, D., \& Croudace, T. J. (2010). An evaluation of the precision of measurement of Ryfff's Psychological Well-Being Scales in a population sample. Social Indicators Research, 97, 357-373. doi:10.1007/s11205-009-9506-x. 
Arnett, J. J. (2016). Introduction: Emerging adulthood theory and research: Where we are and where we should go. In J. J. Arnett (Ed.), The Oxford handbook of emerging adulthood (pp. 1-7). Oxford: Oxford University Press.

Barros-Oliveira, J. H. (2010). Felicidade, optimismo, esperança e perdão em jovens, adultos e idosos. Psychologica, 52 (1), 123148.

Browne, M. W., \& Cudek, R. (1983). Alternate ways of assessing model fit. In K. A. Bollen \& J. S. Long (Eds.), Testing structural equation models (pp. 136-162). Newbury Park, CA: Sage.

Diener, E. (1984). Subjective well-being. Psychological Bulletin, 95, 542-575.

Diener, E. D., Emmons, R. A., Larsen, R. J., \& Griffin, S. (1985). The satisfaction with life scale. Journal of Personality Assessment, 49, 71-75.

Ferreira, J. A. G., \& Jorge, A. (2008). Para a compreensão da adultez emergente em Portugal. Psychologica, 48, 159-173.

Ferreira, J. A., \& Simões, A. (1999). Escalas de Bem-Estar Psicológico (E. B. E. P.). In M. R. Simões, M. M. Gonçalves \& L. S. Almeida (Eds.). Testes e provas psicológicas em Portugal (Vol. 2, pp.111-121). Braga: APPORT/SHO.

Frankl, V. E. (2004). Man's search for meaning (2nd ed.). London: Rider (Obra original publicada em 1946).

Hobfoll, S. E. (1989). Conservation of resources: A new attempt at conceptualizing stress. American Psychologist, 44 (3), 513-524.

Hobfoll, S. E. (2002). Social and psychological resources and adaptation. Review of General Psychology, 6 (4), 307-324.

Holder, M. D., \& Klassen, A. (2009). Personality. In S. J. Lopez (Ed.), The encyclopedia of positive psychology (pp. 689-691). Chichester: Wiley-Blackwell.

Hu, L., \& Bentler, P. M. (1999). Cutoff criteria for fit indexes in covariance structure analysis: Conventional criteria versus new alternatives. Structural Equation Modelling, 6 (1), 1-55.

Huta, V., \& Ryan, R. (2010). Pursuing pleasure or virtue: The differential and overlapping wellbeing benefits of hedonic and eudaimonic motives. Journal of Happiness Studies, 11(6), 735-762.

Iacobucci, D. (2009). Everything you always wanted to know about SEM (Structural Equations Modeling) but were afraid to ask. Journal of Consumer Psychology, 19(4), 673680.

Jahoda, M. (1958). Current concepts of positive mental health. New York: Basic Books.

Laginha, M. C. N. (2015). Otimismo, carreira e bem-estar subjetivo em estudantes do ensino superior (Dissertação de mestrado não publicada). Universidade do Algarve, Faro.

Lopes, J. J. B. (2015). Bem-estar psicológico em estudantes do ensino superior: Relação com as variáveis sociodemográficas, pessoais $e$ académicas (Tese de doutoramento não publicada). Universidade de Évora, Évora.

Machado, W. L. (2010). Escala de Bem-Estar psicológico: Adaptação para o português brasileiro e evidências de validade (Dissertação de mestrado não publicada). Universidade Federal do Rio Grande do Sul, Rio Grande do Sul.

Machado, W. L., \& Bandeira, D. R. (2012). Bemestar psicológico: Definição, avaliação e principais correlatos. Estudos de Psicologia (Campinas), 29 (4), 587-595.

Martins, M., Araújo, A. M., \& Almeida, L. S. (2015). Personalidade e bem-estar subjetivo na excelência profissional: Validação de escalas junto de gestores portugueses. Revista E-Psi, 5(2), 86-102.

Merino, M. D., \& Privado, J. (2015). Positive Psychological Functioning: Evidence for a new construct and its measurement. Anales de Psicología, 31(1), 45-54.

Merino, M. D., Privado, J., \& Gracia, Z. (2015). Validación mexicana de la Escala de Funcionamiento Psicológico Positivo: Perspectivas en torno al estudio del bienestar y su medida. Salud Mental, 38(2), 109-115.

Monteiro, S. O. M. (2008). Optimismo e vinculação na transição para o ensino superior: Relação com sintomatologia psicopatológica, bem-estar e rendimento académico (Tese de doutoramento não publicada). Universidade de Aveiro, Aveiro.

Neto, F., Barros, J., \& Barros, A. (1990). Satisfação com a vida. In L. Almeida, R. 
Santiago, P. Silva, O. Caetano, \& J. Marques (Eds.), A acção educativa: Análise psicosocial (pp.105-117). Leiria: ESEL/APPORT.

Novo, R. F. (2005). Bem-estar e psicologia: Conceitos e propostas de avaliação. Revista Iberoamericana de Diagnóstico y Evaluación - e Avaliação Psicológica, 20(2), 183-203.

Novo, R., Duarte-Silva, E., \& Peralta, E. (1997). O bem-estar psicológico em adultos: Estudo das características psicométricas da versão portuguesa das escalas de C. Ryff. In M. Gonçalves, I. Ribeiro, S. Araújo, C. Machado, L. Almeida, \& M. Simões (Eds.), Avaliação Psicológica: Formas e Contextos (Vol. V, pp. 313 - 323). Braga: APPORT.

Nunnally, J. C., \& Bernstein, I. H. (1994). Psychometric theory (3rd ed.). New York: McGraw-Hill.

Nygren, B., Aléx, L., Jonsén, E., Gustafson, Y., Norberg, A., \& Lundman, B. (2005) Resilience, sense of coherence, purpose in life and self-transcendence in relation to perceived physical and mental health among the oldest old. Aging \& Mental Health, 9(4), 354-362. doi: 10.1080/1360500114415

Porta-Nova, R. M. M. M. (2009). Adaptabilidade, competências pessoais e bem-estar psicológico de jovens do ensino superior na área das Ciências da Saúde (Tese de doutoramento não publicada). Universidade do Porto, Porto.

Raimundi, M. J., Molina, M. F., HernándezMendo, A., \& Schmidt, V. (2017). Adaptación Argentina del Inventario de Fortalezas en Adolescentes (VIA-Youth): Propiedades Psicométricas y Alternativas para su Factorización. Revista Iberoamericana de Diagnóstico y Evaluación - e Avaliação Psicológica, 45(3), 159-174. doi: 10.21865/RIDEP45.3.13

Ramos, L. M. A. (2016). Os mecanismos sóciocognitivos e o bem-estar psicológico: Teste de um modelo integrativo em estudantes universitários (Tese de doutoramento não publicada). Universidade da Beira Interior, Covilhã.

Rodríguez-Carvajal, R., Díaz, D., MorenoJiménez, B., Blanco, A., \& Van Dierendonck, D. (2010). Vitalidad y recursos internos como componentes del constructo de bienestar psicológico. Psicothema, 22(1), 63-70.

doi:10.7764/psykhe.24.2.900

Ryan, R. M., \& Deci, E. D. (2001). On happiness and human potentials: A review of research on hedonic and eudaimonic well-being. Annual Review of Psychology, 52, 141-166.

Ryan, M. R., \& Frederick, C. (1997). On energy, personality, and health: Subjective vitality as a dynamic reflection of well-being. Journal of Personality, 65(3), 529-565.

Ryff, C. D. (1989). Happiness is everything, or is it? Explorations on the meaning of psychological well-being. Journal of Personality and Social Psychology, 57(6), 1069-1081.

Ryff, C. D. (1989). Happiness is everything, or is it? Explorations on the meaning of psychological well-being. Journal of Personality and Social Psychology, 57(6), 1069-1081.

Ryff, C. D. (2016). Beautiful ideas and the scientific enterprise: Sources of intellectual vitality in research on eudaimonic well-being. In J. Vitters $\varnothing$ (Ed.), Handbook of eudaimonic well-being (pp. 95-107). Cham: Springer.

Ryff, C. D., \& Singer B. H. (2008). Know thyself and become what you are: A eudaimonic approach to Psychological Well-Being. Journal of Happiness Studies, 9, 19-39.

Seligman, M. (2012). A vida que floresce: Um novo conceito visionário da felicidade e do bem-estar. Lisboa: Estrela Polar.

Seligman, M. E. P., \& Csikszentmihalyi, M. (2000). Positive psychology: An introduction. American Psychologist, 55, 5-14.

Simões, A. (1992). Ulterior validação de uma escala de satisfação com a vida (SWLS). Revista Portuguesa de Pedagogia, 26, 503515.

Soares, A. P. C. (2003). Transição e adaptação ao ensino superior: Construção e validação de um modelo multidimensional de ajustamento de jovens ao contexto universitário (Tese de doutoramento não publicada). Universidade do Minho, Braga.

Soares, M. L. B. E. S. (2014). Bem-estar psicológico em estudantes do ensino superior: Caracterização, correlatos e proposta de intervenção (Dissertação de mestrado não 
publicada). Universidade Fernando Pessoa, Porto.

Stones, M. J., Worobetz, S., \& Brink, P. (2011). Overestimated relationship with well-being. Canadian Psychology, 52(2), 93-100.

Waterman, A. S. (1984). The psychology of individualism. New York: Praeger.

West, S. G., Finch, J. F., \& Curran, P. J. (1995). Structural equation models with non-normal variables: Problems and remedies. In R. H. Hoyle (Ed.), Structural equation modeling: Concepts, issues and applications (pp. 56-75). Newbury Park, CA: Sage. 
Anexo 1: Escala de Funcionamento Psicológico Positivo (Merino \& Privado, 2015; adaptação portuguesa de Oliveira, Merino, Privado \& Almeida, 2017)

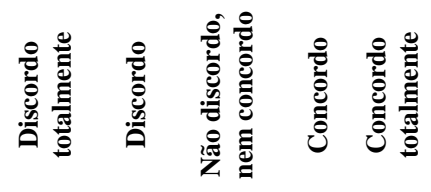

1. Gosto da minha forma de ser.

2. Não me rendo facilmente face às dificuldades da vida.

3. Considero-me uma pessoa otimista.

4. Sei encontrar novos usos para as coisas.

5. Tenho confiança e segurança em mim mesmo/a.

6. Concilio adequadamente a minha vida académica, social e pessoal.

7. Estou cheio/a de vitalidade.

8. As decisões importantes da minha vida, para o melhor ou para o pior, foram tomadas por mim.

9. Sei como relacionar coisas distintas e encontrar algo novo.

10. Eu assumo as rédeas da minha vida.

11. Estou completamente centrado/a em conseguir atingir os objetivos da minha vida.

12. Sou capaz de ver as coisas a partir de pontos de vista completamente diferentes.

13. O sentido de humor é muito importante na minha vida.

14. Perante as dificuldades torno-me forte.

15. Vejo sempre o lado positivo das coisas.

16. Sei desfrutar das pequenas coisas que a vida me oferece em cada dia.

17. Interessa-me tudo o que se passa ao meu redor.

18. Sinto orgulho em ser como sou.

19. Sou uma pessoa entusiasta.

20. Se voltasse a nascer, gostaria de ser tal como sou.

21. Creio que o futuro me trará mais coisas boas que más.

22. Consigo atender a tudo na minha vida diária: amigos, família, estudo,...

23. Sou capaz de rir em muitas situações.

24. Luto para conseguir as coisas que são importantes para mim.

25. Superar dificuldades tornou-me mais forte.

26. Estou bem encaminhado para alcançar os meus objetivos pessoais.

27. Muitas coisas da vida despertam a minha curiosidade e interesse.

28. Sinto-me bem em quase todas as situações.

29. Tento encontrar humor em qualquer situação.

30. Apaixona-me aprender e descobrir coisas novas.

31. Lido com as obrigações que tenho de forma adequada e sem stress.

32. Sou uma pessoa cheia de energia.

33. Na vida há muitas coisas que me enchem de entusiasmo.

\begin{tabular}{|c|c|c|c|c|}
\hline 1 & 2 & 3 & 4 & 5 \\
\hline 1 & 2 & 3 & 4 & 5 \\
\hline 1 & 2 & 3 & 4 & 5 \\
\hline 1 & 2 & 3 & 4 & 5 \\
\hline 1 & 2 & 3 & 4 & 5 \\
\hline 1 & 2 & 3 & 4 & 5 \\
\hline 1 & 2 & 3 & 4 & 5 \\
\hline 1 & 2 & 3 & 4 & 5 \\
\hline 1 & 2 & 3 & 4 & 5 \\
\hline 1 & 2 & 3 & 4 & 5 \\
\hline 1 & 2 & 3 & 4 & 5 \\
\hline 1 & 2 & 3 & 4 & 5 \\
\hline 1 & 2 & 3 & 4 & 5 \\
\hline 1 & 2 & 3 & 4 & 5 \\
\hline 1 & 2 & 3 & 4 & 5 \\
\hline 1 & 2 & 3 & 4 & 5 \\
\hline 1 & 2 & 3 & 4 & 5 \\
\hline 1 & 2 & 3 & 4 & 5 \\
\hline 1 & 2 & 3 & 4 & 5 \\
\hline 1 & 2 & 3 & 4 & 5 \\
\hline 1 & 2 & 3 & 4 & 5 \\
\hline 1 & 2 & 3 & 4 & 5 \\
\hline 1 & 2 & 3 & 4 & 5 \\
\hline 1 & 2 & 3 & 4 & 5 \\
\hline 1 & 2 & 3 & 4 & 5 \\
\hline 1 & 2 & 3 & 4 & 5 \\
\hline 1 & 2 & 3 & 4 & 5 \\
\hline 1 & 2 & 3 & 4 & 5 \\
\hline 1 & 2 & 3 & 4 & 5 \\
\hline 1 & 2 & 3 & 4 & 5 \\
\hline 1 & 2 & 3 & 4 & 5 \\
\hline 1 & 2 & 3 & 4 & 5 \\
\hline 1 & 2 & 3 & 4 & 5 \\
\hline
\end{tabular}

